\title{
Qualitative and Quantitative Predictions of Infectious Diseases in Shirak Marz
}

\author{
Armine Andryan* \\ NCDCP SNCO of the RA Ministry of Health, Yerevan, Armenia
}

\section{Objective}

The goal of this study was to identify the periodicity of seven zooanthroponoses in humans, and set epidemic thresholds for future occurrences.

\section{Introduction}

The frequency of disease outbreaks varies as a result of complex biological processes. Analysis of these frequencies can reveal patterns that can serve as a basis for predictions.

\section{Methods}

A 40 year regression analysis of the following infectious diseases was performed using Arc-GIS10.1 - anthrax, brucellosis, erysipeloid, leptospirosis, plague, tularemia and yersiniosis.

\section{Results}

The analyses covered many years and revealed the dynamics of epidemics for infections. Yearly periodicities of $(3.1+2.8)-(3.8+$ $2.2)$ - 5 - $4.6-2.2-5.1-2.1-2.6$ were determined for theoretically calculated zooanthroponoses. These coincide with the recorded activity of (6 - 6 - 5 - 5 - 2 - 5 - 3 - 2) that correspond to (1977-19831989-1994-1999-2001-2006-2009-2011 (2017-2023-2028). These years had more cases of disease than other years. The predicted years $(2017,2023,2028)$ are those of potential risk, when $0.7-0.9 \%$ of the total disease burden will consist of epidemiologically associated cases.

Disease severity was correlated with natural factors including air temperature, humidity, number of annual heat days, geographical factors, type of landscape, number of carriers, and the contact intensity between disease carrier and transmitter. Partial control indicators (PCIs) were determined to characterize the epidemic situation. These are determined from the perennial average characteristic of the given area from which the mean square deviation is removed. The detection indicator is the normal size of a given disease, with minimal and maximal deviation of the range. It can be compared to the epidemic threshold and helps yield short- and long-term quantitative predictions with high reliability indicators $(96.5 \% \mathrm{p}<0.035)$.

\section{Conclusions}

A 3-5 year periodicity for zooanthroponoses was identified. Conditions contributing to the occurrence of these epidemics differ by region. In Shirak Marz, the PCIs for the different diseases are: brucellosis-47, anthrax-12, plague-8, tularemia-6, leptospirosis-175, erysipeloid-12, yersiniosis-18. These numbers represent years of positive points as a maximum threshold. The stability index was identified, for instance, for brucellosis $\mathrm{S}=1.2$, amplitude - 5.2, perennial average - 28.8, orientation month - January, seasonal morbidity ratio - 18-42 cases. Our predictions indicate that 2017 will be a peak year with $95 \%$ probability; intensive index: 16.8 (per 100,000 population), seasonal illness cases: $42 \pm 3.5$ between March and November. The application of numerical thresholds in predictive epidemiological surveillance provide clear triggers that make public health responses more targeted and rational.

\section{Keywords}

regression analysis; periodicity; predictive epidemiological surveillance

\author{
*Armine Andryan \\ E-mail: anarmine@mail.ru
}

